\title{
Study of the Lunar Core by VLBI Observations of Artificial Radio Sources on the Moon
}

\author{
Hideo HANAdA ${ }^{1}$, Masatsugu OoE $^{1}$, Noriyuki KaWAGUCHI ${ }^{2}$, Nobuyuki Kawano ${ }^{1}$, Seisuke KuJI ${ }^{1}$, \\ Tetsuo SASAO ${ }^{1}$, Seiitsu Tsuruta ${ }^{1}$, Mitsumi Fujishita ${ }^{3}$, and Masaki Morimoto ${ }^{2 *}$ \\ ${ }^{1}$ Mizusawa Astrogeodynamics Observatory/National Astronomical Observatory, \\ 2-12 Hoshigaoka, Mizusawa, Iwate 023, Japan \\ ${ }^{2}$ Division of Radio Astronomy, National Astronomical Observatory, \\ 411 Nobeyama, Minamimakimura, Minamisakugun, Nagano 384-13, Japan \\ ${ }^{3}$ School of Engineering, Kyushu Tokai University, 9-1-1 Toroku, Ooe, Kumamoto, Kumamoto 862, Japan
}

(Received December 4, 1992; Revised August 4, 1993; Accepted August 19, 1993)

\begin{abstract}
Differential VLBI technique can measure the variation in the angular distance between two artificial radio transmitters on the Moon with an accuracy of a few centimeters. We can observe the physical librations of the Moon through this technique since rotation of the Moon changes the angular distance. The gravitational field of the Moon can also be determined by observations of the angular distance between the radio source on the Moon and that on a lunar orbiter. Calculations show that this technique can determine the amplitude of the physical librations with an accuracy of $10^{-5}$ which are one or two orders higher than those obtained by using the Apollo Lunar Surface Experiments Package in 1970's. We can estimate the density and the radius of the lunar core through accurate amplitude and period of the librations together with seismic observations. The accuracy of determination of the lunar gravity harmonics of degree 2 derivable from the VLBI observations are in the range from $10^{-4}$ to $10^{-6}$. We are developing radio transmitters which will be put on the lunar surface by hard landing from the lunar orbiter.
\end{abstract}

\section{Introduction}

It is very important to know the radius and the mass of the lunar core in order to investigate the origin and the evolution of the Moon, since they can put a constraint on the composition and the formation of the Moon. NAKAmURA et al. (1974) estimated the radius of the lunar core to be from 170 to $360 \mathrm{~km}$ by observing $\mathrm{P}$ waves generated by a single backside impact. The moment of inertia, which can be obtained by observations of the amplitude of the physical librations of the Moon, is also related to the radius of the core, and BILL and FERRARI (1977) proposed a model consistent with the mean moment of inertia estimated from low-degree gravity harmonics and librational parameters by assuming a core of radius $340 \mathrm{~km}$ and of density $5.2 \times 10^{3} \mathrm{~kg} / \mathrm{m}^{3}$. The value $C / M R^{2}$, where $C$ is the lunar maximum moment of inertia, $M$ the lunar mass and $R$ the lunar mean radius, probably lies in the range between 0.390 and 0.393 , and the uncertainty of it is equivalent to the uncertainty of the radius of the lunar core of about $100 \mathrm{~km}$ (BINDER, 1980). It is necessary to improve the accuracy of determination of the lunar moment of inertia in order to put a strong constraint on the radius of the core.

The period of the free librations and the Love's numbers of the Moon are also considered to be functions of the radius of the lunar core. HiLton (1992) showed that the period of the free-core nutation (FCN) is very sensitive to the core radius in the Mars' models. Observation of the period of the FCN will be effective also for estimation of the radius of the lunar core if

*Present address: Faculty of Liberal Arts, University of Kagoshima, 1-21-30 Korimoto, Kagoshima, Kagoshima 890, Japan. 
it is liquid. The existence of a liquid core in the Moon is suggested by the lunar laser ranging (YODER, 1981), observations of the lunar magnetic fields (HoOD et al., 1978), and geochemical investigations (NEWSOM and TAYLOR, 1989). ZHANG (1992), on the other hand, showed that there are simple relations between the second degree Love number and the lunar core radius by means of lunar models consistent with the mean moment of inertia and the mean density of the Moon. The Love number can be estimated by observations of lunar tides.

We need to improve the accuracy of determination of the lunar moment of inertia and that of the period of the free libration of the Moon in order to put stronger constraints on the radius of the lunar core. VLBI (Very Long Baseline Interferometry), which is one of the most sensitive geodetic technique, can measure the distance between two continents with a few centimeter accuracy (HEKI et al., 1990) and can measure the direction of the Earth's spin axis to better than milli-arc-second accuracy (HERRING et al., 1991). These 'geodetic' VLBI observations measure group delays by means of the bandwidth synthesis technique. It is well known that the fringe phase difference observable is much more sensitive to the variation of the angular distance than the group delay observable (see, for example, THOMPSON et al., 1986). The accuracy becomes still better when we measure the angular distance between two radio sources which are close to each other since the destructive effects of excess path delays in the ionosphere and the troposphere as well as the fluctuations in the frequency standard are almost equal and are canceled in the differential observation. This technique is called differential VLBI in terms of the fringe-phase-difference measurements (Counselman et al., 1973; MARCAIDE and ShAPIRO, 1983; BARTEL et al., 1986; GWINn et al., 1986; LESTRADE et al., 1990). Figure 1 shows the outline of the differential VLBI observations using radio sources on the Moon and the lunar orbiter. Each of the fringe phases is tracked as long as the radio sources are in common view. The angular distance between the two radio sources can be derived from the difference of their fringe phases. It will be possible to measure the change in distance between two radio sources on the Moon with an accuracy of a few centimeters by using the differential VLBI (KAWANO et al., 1992). Then we can expect highly accurate measurements of lunar rotation and lunar deformation.

The first experiment by using VLBI and artificial radio sources on the Moon was performed as a part of the Apollo project in the 1970s, and the principal moments of inertia and low degree

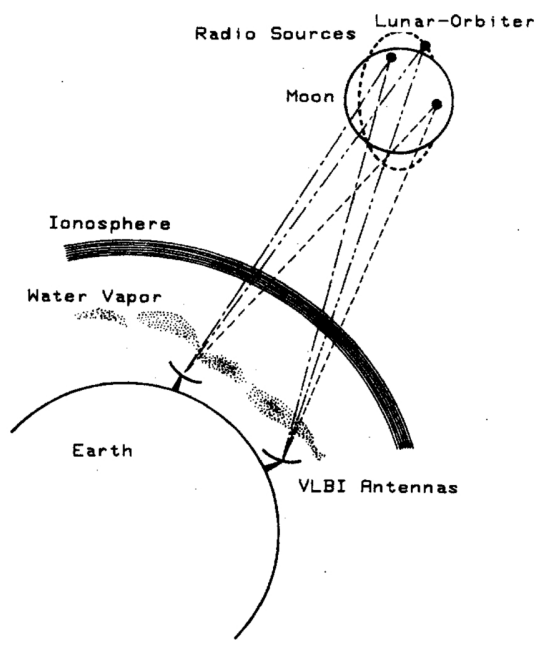

Fig. 1. A diagram showing the differential VLBI using radio sources on the Moon and a lunar orbiter. 
gravity harmonics were determined with uncertainties of from $10^{-4}$ to $10^{-5}$ and from $10^{-1}$. to $10^{-2}$, respectively (KING et al., 1976). The accuracy of the VLBI at that time, however, was about 2 orders of magnitude lower than the current accuracy. There would be great improvement in selenodetic data if we carry out such an experiment now. Institute of Space and Astronautical Science (ISAS) is pursuing the plan to put seismometers and heat-flow-meters on the Moon by using penetrators (MIZUTANI et al., 1990) and we are developing radio transmitters for VLBI which can emit $2.2 \mathrm{GHz}$ sinusoidal waves for more than one year with batteries after surviving the strong shock at the time of its penetration into the lunar surface.

In this paper, we describe the expected accuracy of determination of the period of the free librations, the amplitude of the forced librations, the harmonics of the lunar gravitational field, etc. derived by the differential VLBI observations of the radio transmitters on the lunar surface and the orbiter, and describe the information on the lunar core which will be obtained by the observations.

\section{Observation of the Librations}

There are physical libration and the optical libration in the lunar librations. The optical libration is apparent periodic oscillation in longitude or in latitude caused by geometrical effect of the eccentricity and the inclination of the lunar orbit and has no physical information on the Moon. The physical libration, on the other hand, is due to Earth's periodic gravitational torque, lunar eccentric orbit and lunar asymmetric mass distribution and behaves like the Earth's nutation. We focus on the physical libration since it is related to physical parameters of the Moon such as moment of inertia. The physical libration consists of forced libration and free libration corresponding to the Earth's nutation and the Chandler wobble, respectively. It has three components, that are inclination $(i)$, node $(\omega)$, and longitude $(\Omega)$, respectively, and each component is described by rotation around the axes $x, z$ and $Z$, respectively, where $x y$ plane is the lunar equator (see Fig. 2). For simplicity, if we suppose that the $X$-axis points in the direction of the Earth and the $Z$-axis is perpendicular to the ecliptic, we can observe change of distance only on the $Y Z$ plane with VLBI.

When the inclination $i$ changes by $\Delta i$, displacements of a radio source on the Moon for the directions of $Y$ and $Z$-axes are respectively expressed as

$$
\begin{gathered}
\Delta Y=[(x \sin \omega+y \cos \omega) \cos \phi \sin i+z \cos \phi \cos i] \Delta i, \\
\Delta Z=[(x \sin \omega-y \cos \omega) \cos i-z \sin i] \Delta i
\end{gathered}
$$

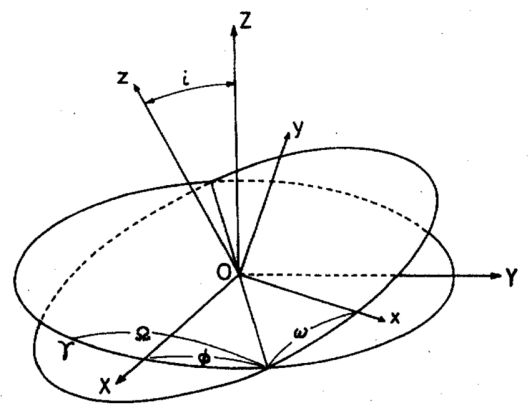

Fig. 2. A coordinate system fixed on the Moon $(x y z)$ and the inertial reference system $(X Y Z)$. 
when the longitude of the ascending node $\Omega$ changes by $\Delta \Omega$, they are

$$
\begin{gathered}
\Delta Y=[x(\sin \phi \sin \omega \cos i-\cos \phi \cos \omega)-y(\sin \phi \cos \omega \cos i+\cos \phi \sin \omega)-z \sin \phi \sin i] \Delta \Omega, \\
\Delta Z=0
\end{gathered}
$$

and when the angle between the ascending node and the direction of $x$-axis $\omega$ changes by $\Delta \omega$, they are

$$
\begin{gathered}
\Delta Y=[x(\sin \phi \sin \omega-\cos \phi \cos \omega \cos i)-y(\sin \phi \cos \omega+\cos \phi \sin \omega \cos i)] \Delta \omega, \\
\Delta Z=(x \cos \omega \sin i+y \sin \omega \sin i) \Delta \omega,
\end{gathered}
$$

where $\phi$ is the angle between $X$-axis and the ascending node (see Fig. 2). If we approximate $i \approx 0, \phi \approx 0$ and $\omega \approx 0$, Eqs. (1)-(6) then become

$$
\begin{array}{cc}
\Delta Y=z \Delta i, & \Delta Z=-y \Delta i, \\
\Delta Y=-x \Delta \Omega, & \Delta Z=0, \\
\Delta Y=-x \Delta \omega, & \Delta Z=0 .
\end{array}
$$

Since we can measure the displacement only for the directions of $Y$ and $Z$ axes, observed change of the distance between two radio sources by differential VLBI is expressed as

$$
\Delta l=\sqrt{\left(\Delta Y_{2}-\Delta Y_{1}\right)^{2}+\left(\Delta Z_{2}-\Delta Z_{1}\right)^{2}},
$$

where the subscripts 1 and 2 indicate the radio source numbers. The expected colatitudes and longitudes of the penetrators thrown from the Lunar- $\mathrm{A}$ are $\left(0^{\circ}, 0^{\circ}\right)$ and $\left(95^{\circ},-35^{\circ}\right)$, respectively (FUjIMURA, 1991). The positions of them in $x y z$ coordinate are $(0,0, R)$ and $(0.82 R,-0.57 R$, $-0.09 R)$. If we suppose that the radio sources are at the same positions of the penetrators, we can estimate change of the distance $\Delta l$ by using Eqs. (7)-(10) and we get $\Delta l=1.23 R \Delta i$, $\Delta l=0.82 R \Delta \Omega$ and $\Delta l=0.82 R \Delta \omega$. The maximum change of the distance $\Delta l$ will be about 700 $\mathrm{m}$ for the librations in node and longitude and about $1 \mathrm{~km}$ for the libration in inclination since the maximum amplitude of any component of the physical libration is estimated to be about 100 arc second (ECKHARDT, 1981). Accuracy of determination of the amplitude of the physical libration then will be about $10^{-5}$ if the accuracy of the differential VLBI for measurement of the displacement on the lunar surface is $1 \mathrm{~cm}$.

The amplitude of the physical libration is dependent upon both the low-degree gravity harmonics and the parameters $\alpha, \beta$ and $\gamma$ which are respectively defined as $\alpha=(C-B) / A$, $\beta=(C-A) / B, \gamma=(B-A) / C$ with lunar principal moments of inertia $A<B<C$. Let the direction cosines of the $Z$-axis (pole of the ecliptic) referenced to the moon fixed axes $x, y$, and $z$ be $p_{1}, p_{2}$, and $p_{3}$, respectively. These are conventionally used as variables representing the physical librations and they are related to the Euler angles as

$$
\left[\begin{array}{l}
p_{1} \\
p_{2} \\
p_{3}
\end{array}\right]=\left[\begin{array}{c}
\sin i \sin \omega \\
\sin i \cos \omega \\
\cos i
\end{array}\right] .
$$

A first-order equations for the physical librations in terms of the variables $p_{1}, p_{2}$ and $\Delta \omega$ are

$$
\begin{gathered}
\ddot{p}_{1}-N \dot{p}_{2}(1-\beta)+4 \beta N^{2} P_{1}=-3 \beta N^{2} \sin I \sin F, \\
\ddot{p}_{2}+N \dot{p}_{1}(1-\alpha)+\alpha N^{2} P_{2}=0,
\end{gathered}
$$




$$
\Delta \ddot{\omega}+3 \gamma N^{2} \Delta \omega=3 \gamma N^{2} \sin \left(l^{\prime}-l\right),
$$

where $I$ is inclination of the Moon's orbit to the ecliptic, $l^{\prime}$ true longitude of the Moon, $l$ mean longitude of the Moon, $F$ mean argument of the latitude of the Moon $(F=l-\Omega)$ and $N=i=$ $1 / 27.322$ day $^{-1}$, respectively. The particular solutions of (12)-(14) are

$$
\left[\begin{array}{c}
p_{1} \\
p_{2} \\
\Delta \omega
\end{array}\right]=\left[\begin{array}{c}
3 \beta \sin I \sin F /(2 \mu-3 \beta) \\
3 \beta \sin I \cos F /(2 \mu-3 \beta) \\
{\left[1-\left(\dot{l}^{\prime}-\dot{l}\right)^{2} /\left(3 \gamma N^{2}\right)\right]^{-1} \sin \left(l^{\prime}-l\right)}
\end{array}\right]
$$

with $\dot{F} / N=1+\dot{\Omega} / N=1+\mu$ (ECKHARDT, 1981). The amplitude of the physical librations are thus proportional to the parameters $\alpha, \beta$ and $\gamma$, and the accuracies in the estimation of the parameters will be almost the same as those in the amplitudes of the librations. For a rigid Moon, there are relationships among the parameters, the lunar moment of inertia and the second degree gravity harmonics:

$$
C / M R^{2}=4 C_{22} / \gamma
$$

and

$$
\frac{I}{M R^{2}}=\frac{4 C_{22}(3+\beta+\gamma-\beta \gamma)}{3 \gamma(1+\beta)}
$$

where $I(I=(A+B+C) / 3)$ is the mean moment of inertia (FERRARI et al., 1980). We expect accuracy of determination of the maximum lunar moment of inertia $C / M R^{2}$ to be better than $10^{-5}$ by the measurement of the amplitudes of the physical librations and the gravity harmonics $C_{22}$ with $10_{-}^{-5}$ accuracy.

The moments of inertia, on the other hand, reflect density distribution of the Moon and we can put a constraint upon the radius and the density of the lunar core from them if we know a mean density in a mantle (the region outside the core). ANANDA et al. (1977) showed how the amplitude of the physical libration is sensitive to the density of the lunar core. According to their result, sensitivity of $10^{-4}$ in the amplitude of the physical libration is equivalent to that of 0.1 $\mathrm{gr} / \mathrm{cm}^{3}$ in estimation of density of the lunar core if the radius of the core is $400 \mathrm{~km}$. Figure 3 shows the relation between uncertainty in density of the lunar core and radius of the lunar core in the case that the lunar mean moment of inertia is observed with $10^{-4}$ accuracy. In this figure, the mean density of the whole Moon and the mean moment of inertia are assumed to be precisely known as $3.3437 \times 10^{3} \mathrm{~kg} / \mathrm{m}^{3}$ and 0.3922 , respectively.

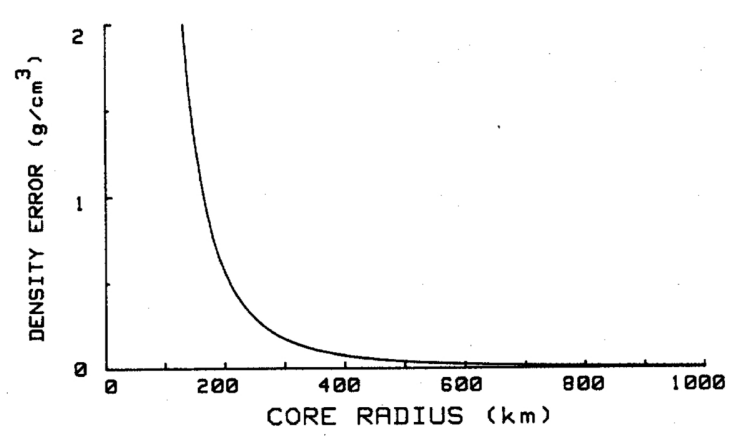

Fig. 3. Uncertainty in the density of the lunar core for assumed core radius in the case that the lunar moment of inertia ratio is observed with $10^{-4}$ accuracy. 
It is usually impossible to separately know the mean density of the core and that of the mantle from only the moments of inertia of the whole Moon. If the Moon has a spherical liquid core and if the core-mantle boundary is frictionless, we can substitute the parameters $\alpha, \beta$ and $\gamma$ in Eq. (15) by $\alpha^{\prime}=(C-B) /\left(A-I_{C}\right), \beta^{\prime}=(C-A) /\left(B-I_{C}\right)$ and $\gamma^{\prime}=(B-A) /\left(C-I_{C}\right)$, where $I_{C}$ is the mean moment of inertia of the core. Then we may obtain information of the moment of inertia of the core alone from the parameters $\alpha^{\prime}, \beta^{\prime}$ and $\gamma^{\prime}$ obtained by the observation of the physical libration. The moments of inertia $A, B$ and $C$ must be precisely known from other sources for this purpose.

The free libration of the Moon, on the other hand, has sensitivity for radius of the lunar core in its period. CALAME (1977) analyzed the lunar laser ranging data for six years and got the values of $0^{\prime \prime} .4,7^{\prime \prime} .8$ and $1^{\prime \prime} .8$ as the amplitudes of the three components of the free librations with the respective periods of about 27.3 days, 75 years and 2.9 years. YoDER (1981) has also detected the free librations whose amplitude are equivalent to those of CALAME (1977) by lunar laser ranging. However, number of observations of the free librations made so far is very small and there has not yet been obtained reliable values for the amplitude and the period of them.

The equations for the free librations for a rigid Moon are obtained from Eqs. (12)-(14). Let the three components of the free libration be written as $p_{1}=P_{1} \sin \left(\sigma_{1} t+\delta_{1}\right), p_{2}=P_{2} \cos \left(\sigma_{2} t+\delta_{2}\right)$ and $\Delta \omega=\Delta \omega_{0} \sin \left(\sigma_{3} t+\delta_{3}\right)$. Then they satisfy the relations

$$
\begin{gathered}
\ddot{p}_{1}-N \dot{p}_{2}(1-\beta)+4 \beta N^{2} P_{1}=0, \\
\ddot{p}_{2}+N \dot{p}_{1}(1-\alpha)+\alpha N^{2} P_{2}=0, \\
\Delta \ddot{\omega}+3 \gamma N^{2} \Delta \omega=0 .
\end{gathered}
$$

The eigenfrequencies of Eqs. (18)-(20) are $\sigma_{1}= \pm(1+3 \beta / 2) N, \sigma_{2}= \pm 2 N \sqrt{\alpha \beta}, \sigma_{3}=N \sqrt{3 \gamma}$ (LAMBECK, 1988). If we substitute the observed values of the parameters $\alpha=(4.036 \pm 0.007) \times$ $10^{-4}, \beta=(6.313 \pm 0.0003) \times 10^{-4}$ and $\gamma=(2.277 \pm 0.007) \times 10^{-4}$ (KING et al. 1976), the eigenfrequencies become $\sigma_{1}=1 / 27.3$ day $^{-1}, \sigma_{2}=1 / 27064$ day $^{-1}$ and $\sigma_{3}=1 / 1045.4$ day $^{-1}$.

If there is a liquid core in the Moon, the eigenfrequencies of the free libration will be affected by the change in the moment of inertia and the core-mantle coupling. The first order effect of the liquid core on the free libration is obtained by assuming that the core is spherical and that the core-mantle boundary is frictionless. Then the eigenfrequencies of the free libration with a liquid core are obtained by respectively substituting the parameters $\alpha, \beta$ and $\gamma$ by $\alpha^{\prime}, \beta^{\prime}$ and $\gamma^{\prime}$ in the same manner as the physical librations. In the case that the radius of the liquid core is

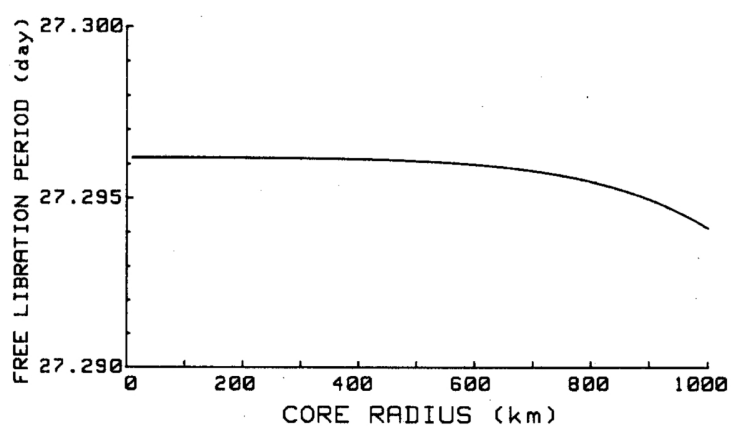

Fig. 4. Relation between the period of one component of the lunar libration and the radius of the lunar core filled with liquid. 
$450 \mathrm{~km}$ and the density is $7.9 \times 10^{3} \mathrm{~kg} / \mathrm{cm}^{3}$, change of the eigenfrequencies $\Delta \sigma_{1}, \Delta \sigma_{2}$ and $\Delta \sigma_{3}$ become $\Delta \sigma_{1} / \sigma_{1}=2.87 \times 10^{-6}, \Delta \sigma_{2} / \sigma_{2}=3.04 \times 10^{-3}$ and $\Delta \sigma_{3} / \sigma_{3}=1.54 \times 10^{-3}$, respectively. The corresponding changes in period are $\Delta T_{1}=6.7 \mathrm{~s}, \Delta T_{2}=82.3$ days and $\Delta T_{3}=1.6$ days. It is possible to detect these changes by the VLBI observations. Figure 4 shows the relation between the eigenperiod $T_{1}$ and the radius of the liquid core under the condition where the mass of the whole Moon is kept constant.

\section{Determination of the Lunar Gravitational Field}

The moment of inertia of the Moon cannot be derived solely from observations of the physical librations. Low degree gravity harmonics of the Moon are necessary in conjunction with the amplitudes of the physical librations to provide the lunar moment of inertia. We therefore should know them with the accuracy equivalent to that of the physical librations. The lunar gravitational fields are usually determined by Doppler tracking of artificial lunar satellites. It is also possible to determine the gravitational fields by the VLBI observations if we load a radio transmitter in a lunar satellite and if we observe the angular distance between the radio transmitter in the satellite and that on the lunar surface or radio stars. The Doppler tracking has the maximum sensitivity in the direction along the mean Earth-Moon line and the VLBI observation, on the other hand, has one in the direction perpendicular to it. Although combination of the two different method will bring about the best result, we estimate here the accuracy of the lunar gravitational field determined by only the VLBI observation.

Let, for example, the initial orbital elements of the lunar satellite be $a=1840 \mathrm{~km}, i=90$ deg., $e=0.1, \Omega=0$ deg., $\omega=0$ deg., where $a$ is semi-major axis, $i$ inclination, $e$ eccentricity, $\Omega$ longitude of ascending node and $\omega$ argument of perigee, respectively (KAULA, 1966). The changing rate of the longitude of ascending node $\dot{\Omega}$ and that of the mean anomaly $\dot{M}$ can be observables of the VLBI, where $M$ is defined by

$$
M=N_{0}\left(T-T_{P}\right)
$$

with the mean angular velocity $N_{0}$ and the time at which the satellite passes $T_{P}$. Since the period of the lunar satellite is about $120 \mathrm{~min}$., the observation accuracy of $\dot{\Omega}$ and $\dot{M}$ is about $1 / 120 \mathrm{~cm} / \mathrm{min}$. in velocity or $7.55 \times 10^{-13} \mathrm{rad} / \mathrm{s}$ in angular velocity at the best with the accuracy of the differential VLBI of $1 \mathrm{~cm}$. The factor $\cos \theta$ is multiplied if the direction of the motion has angle $\theta$ to the $Y Z$ plane. The accuracies of $\dot{\Omega}$ and $\dot{M}$ are functions of position of the satellite and they are written as

$$
\begin{gathered}
\Delta \dot{\Omega}=\Delta \dot{\Omega}_{0} \cos \theta \cos M, \\
\Delta \dot{M}=\Delta \dot{M}_{0}(1-\cos \theta \sin M),
\end{gathered}
$$

where $\theta$ is hour angle of the ascending node and $\Delta \dot{\Omega}_{0}=\Delta \dot{M}_{0}=7.55 \times 10^{-13} \mathrm{rad} / \mathrm{s}$.

Time variation of the orbital elements of the satellite can be expanded with the series of spherical harmonic coefficients of the lunar gravitational field (KAULA, 1966). The expansion of the changing rate of the longitude of ascending node $\dot{\Omega}$ and that of the mean anomaly $\dot{M}$ with the spherical harmonic coefficients through degree and order 4 are written as

$$
\begin{aligned}
\dot{\Omega}= & +1.42 \times 10^{-3}\left(C_{21} \sin \theta+S_{21} \cos \theta\right) \\
& -2.44 \times 10^{-3}\left(C_{41} \sin \theta+S_{41} \cos \theta\right) \\
& -3.45 \times 10^{-2}\left(C_{43} \sin 3 \theta+S_{43} \cos 3 \theta\right),
\end{aligned}
$$




$$
\begin{aligned}
\dot{M}= & +5.97 \times 10^{-4} C_{20} \\
& +4.23 \times 10^{-3}\left(C_{22} \cos 2 \theta-S_{22} \sin 2 \theta\right) \\
& -2.71 \times 10^{-3}\left(C_{31} \cos \theta-S_{31} \sin \theta\right) \\
& -9.59 \times 10^{-2}\left(C_{33} \cos 3 \theta-S_{33} \sin 3 \theta\right) \\
& +4.29 \times 10^{-4} C_{40} \\
& +6.72 \times 10^{-3}\left(C_{42} \cos 2 \theta-S_{42} \sin 2 \theta\right) \\
& -6.35 \times 10^{-2}\left(C_{44} \cos 4 \theta-S_{44} \sin 4 \theta\right) .
\end{aligned}
$$

The ascending node of the satellite moves relative to the Moon with an angular velocity of about $2.66 \times 10^{-6} \mathrm{rad} / \mathrm{s}$ due to the lunar rotation and it rotates around the Moon by about 180 degrees for 165 revolutions since the period of the satellite is about 120 minutes. We consider the following three types of VLBI observations of the satellite.

Type I: the VLBI observes the position of the satellite in every revolution only when it passes the lunar equator,

Type II: the VLBI observes the position when the satellite passes $-60^{\circ}$ in the first revolution, $0^{\circ}$ in the second one, $+60^{\circ}$ in the third one and continues this sequence,

Type III: the VLBI observes the position when the satellite passes the three latitudes of $-60^{\circ}, 0^{\circ}$ and $+60^{\circ}$ in every revolution.

The number of observations for two components $\dot{\Omega}$ and $\dot{M}$ becomes 330 as to the types I and II, and 990 as to the type III. Table 1 shows the estimated accuracy of the coefficients of the fourth degree and order for the lunar gravitational field determined by the least square method with the probable data obtained by the VLBI observations of types I, II and III supposing that the VLBI observes the satellite position with the accuracy of $1 \mathrm{~cm}$. The values shown in Table 1

Table 1. Accuracy of lunar gravity harmonics.

\begin{tabular}{llll}
\hline Harmonics & I. $(n=330)$ & II. $(n=330)$ & III. $(n=990)$ \\
\hline$C_{20}$ & $3.33 \times 10^{-6}$ & $4.73 \times 10^{-6}$ & $1.75 \times 10^{-6}$ \\
$C_{21}$ & $3.22 \times 10^{-4}$ & $7.73 \times 10^{-3}$ & $4.43 \times 10^{-4}$ \\
$C_{22}$ & $2.96 \times 10^{-5}$ & $1.07 \times 10^{-5}$ & $2.54 \times 10^{-6}$ \\
$C_{30}$ & & & \\
$C_{31}$ & $2.26 \times 10^{-6}$ & $2.08 \times 10^{-6}$ & $4.21 \times 10^{-6}$ \\
$C_{32}$ & & & \\
$C_{33}$ & $1.11 \times 10^{-9}$ & $4.35 \times 10^{-8}$ & $3.78 \times 10^{-9}$ \\
$C_{40}$ & $6.66 \times 10^{-5}$ & $3.80 \times 10^{-5}$ & $1.08 \times 10^{-4}$ \\
$C_{41}$ & $1.59 \times 10^{-6}$ & $1.02 \times 10^{-4}$ & $4.77 \times 10^{-6}$ \\
$C_{42}$ & $2.45 \times 10^{-5}$ & $6.94 \times 10^{-5}$ & $1.95 \times 10^{-5}$ \\
$C_{43}$ & & & \\
$C_{44}$ & $5.38 \times 10^{-9}$ & $4.28 \times 10^{-7}$ & $1.56 \times 10^{-7}$ \\
\hline$S_{20}$ & & & \\
$S_{21}$ & & & \\
$S_{22}$ & $3.53 \times 10^{-3}$ & $2.69 \times 10^{-3}$ & $4.87 \times 10^{-4}$ \\
$S_{30}$ & & & \\
$S_{31}$ & $8.92 \times 10^{-7}$ & $5.27 \times 10^{-7}$ & $1.28 \times 10^{-6}$ \\
$S_{32}$ & & & \\
$S_{33}$ & $5.34 \times 10^{-7}$ & $3.23 \times 10^{-7}$ & $3.99 \times 10^{-7}$ \\
$S_{40}$ & & & \\
$S_{41}$ & $1.29 \times 10^{-4}$ & $6.41 \times 10^{-5}$ & $1.61 \times 10^{-4}$ \\
$S_{42}$ & $2.25 \times 10^{-5}$ & $1.67 \times 10^{-5}$ & $3.84 \times 10^{-6}$ \\
$S_{43}$ & & & \\
$S_{44}$ & $3.63 \times 10^{-6}$ & $1.97 \times 10^{-6}$ & $1.40 \times 10^{-6}$ \\
\hline & & & \\
& & &
\end{tabular}


are normalized with the respective spherical harmonic coefficients of the lunar gravitational field obtained by BILL and FERRARI (1980). It will be possible to determine the spherical harmonic coefficients of degree 2 , which are related to the lunar moments of inertia, with the accuracy of better than $10^{-6}$ except $C_{21}$ and $S_{22}$.

\section{Concluding Remarks}

We showed that the differential VLBI technique can measure the amplitudes of the physical librations with the sensitivity of about $10^{-5}$ and the spherical harmonic coefficients of the lunar gravitational field of degree 2 with the sensitivity of $10^{-6}$. We also showed that the highly sensitive data of the physical librations are important to estimate the radius, the density and the condition of the lunar core. It is necessary to observe over a long time of at least one year in order to separate the similar terms included in the physical librations. We are now successfully developing artificial radio sources which will be set on the lunar surface from the lunar orbiter by hard landing. The preliminary experiment showed that the radio sources succeeded to emit the signal after the impact of about $10^{4} \mathrm{~g}$. The most important problem is to improve the efficiency of power consumption of the radio sources for long-term observations.

The authors would like to express their appreciation to Drs. H. Mizutani and A. Fujimura of the Institute of Space and Astronautical Science, Drs. O. Kameya, K.-H. Sato, Y. Tamura, T. Hara and S. Yasuda of the National Astronomical Observatory, Mizusawa, and Dr. D. Stephenson of the University of California for helpful comments and discussions.

\section{REFERENCES}

Ananda, M. P., A. J. Ferrari, and W. L. SJogren, An improved lunar moment of inertia determination: a proposed strategy, Moon, 17, 101-120, 1977.

Bartel, N., T. A. Herring, M. I. Ratner, I. I. Shapiro, and B. E. Corey, VLBI limits on the proper motion of the 'core' of the superluminal quasar 3C345, Nature, 319, 733-738, 1986.

Bill, B. G. and A. J. FerrarI, A lunar density model consistent with topographic, gravitational, librational, and seismic data, J. Geophys. Res., 82, 1306-1314, 1977.

Bill, B. G. and A. J. Ferrari, A harmonic analysis of lunar gravity, J. Geophys. Res., 85, 1013-1025, 1980.

Binder, A. B., On the internal structure of a moon of fission origin, J. Geophys. Res., 85, 4872-4880, 1980.

Calame, O., Free librations of the moon from lunar laser ranging, in Scientific Applications of Lunar Laser Ranging, edited by J. D. Mulholland, pp. 53-63, D. Reidel, Dordrecht, 1977.

Counselman, C. C., III, H. F. Hinteregger, R. W. King, and I. I. Shapiro, Precision selenodesy via differential interferometry, Science, 181, 772-774, 1973.

Eckhardt, D. H., Theory of the libration of the moon, Moon and Planets, 25, 3-49, 1981.

Ferrari, A. J., W. S. Sinclair, W. L. Sjogren, J. G. Williams, and C. F. Yoder, A low degree lunar gravity model from combined Doppler and laser ranging data, J. Geophys. Res., 85, 3939-3951, 1980.

Fujimura, A., Seismological exploration on extraterrestrial bodies, Zisin, J. Seism. Soc. Japan, 44, 41-52, 1991 (in Japanese).

Gwinn, C. R., J. H. TAYlor, J. M. Weisberg, and L. A. RaWley, Measurement of pulsar parallaxes by VLBI, Astron. J., 91, 338-342, 1986.

HEKI, K., Y. TAKAHASHI, and T. KoNDo, Contraction of northeastern Japan: evidence from horizontal displacement of a Japanese station in global very long baseline interferometry networks, Tectonophysics, 181, 113-122, 1990.

Herring, T. A., B. A. Buffett, P. M. Mathews, and I. I. Shapiro, Forced nutations of the Earth: Influence of inner core dynamics 3. Very long interferometry data analysis, J. Geophys. Res., 96, 8259-8273, 1991.

Hilton, J. L., The motion of Mars' pole. II. The effect of an elastic mantle and a liquid core, Astronom. J., 103, 619-637, 1992.

Hood, L., C. T. Russell, and P. J. Coleman, Jr., Evidence for a non-random magnetization of the moon, Geophys. Res. Lett., 5, 305-308, 1978.

KaUla, W. M., Theory of Satellite Geodesy, Blaisdell, Waltham, Mass, 1966.

Kawano, N., S. Kuji, H. Hanada, S. Tsuruta, T. SASAo, and N. Kawaguchi, Investigations on a lunar transmitter, Technical Report of Mizusawa Kansoku Center, Nat. Astro. Obs., 4, 116-127, 1992 (in Japanese).

King, R. W., C. C. Counselman, III, and I. I. Shapiro, Lunar dynamics and selenodesy: results from analysis of VLBI and laser data, J. Geophys. Res., 81, 6251-6256, 1976. 
LAmbeCK, K., Geophysical Geodesy, pp. 330-352, Clarendon Press, Oxford, 1988.

Lestrade, J.-F., A. E. E. Rogers, A. R. Whitney, A. E. Niell, R. B. Phillips, and R. A. Preston, Phasereferenced VLBI observations of weak radio sources, milliarcsecond position of Algol, Astron. J., 99, 1663-1673, 1990.

MARCAIDE, J. M. and I. I. SHAPIRO, High precision astrometry via very-long-baseline radio interferometry: estimate of the angular separation between the quasars 1038+528A and B, Astron. J., 88, 1133-1137, 1983.

Mizutani, H., A. Fujimura, N. Kawashima, T. Tanaka, and I. Yamada, Scientific significance of the lunar exploration by using penetrators, Proc. 13th Symposium on the Solar System, 18-23, 1990 (in Japanese).

Nakamura, Y., G. Latham, D. Lammlein, M. Ewing, F. Duennebier, and J. Dorman, Deep interior inferred from recent seismic data, Geophys. Res. Lett., 1, 137-140, 1974.

Newsom, H. E. and S. R. TAYLOR, Geochemical implications of the formation of the Moon by a single giant impact, Nature, 338, 29-34, 1989.

Thompson, A. R., J. M. Moran, and G. W. Swenson, Jr., Interferometry and Synthesis in Radio Astronomy, John Wiley, New York, 1986.

Yoder, C. F., The free librations of a dissipative Moon, Phil. Trans. R. Soc. Lond., A303, 327-338, 1981.

ZHANG, C. Z., Love numbers of the Moon and of the terrestrial planets, Earth, Moon, Planets, 56, $193-207,1992$. 